\title{
Alterações oculares em crianças pré-escolares e escolares no município de Duque de Caxias, Rio de Janeiro, Brasil
}

\author{
Eye diseases in preschool and school children in the city of \\ Duque de Caxias, Rio de Janeiro, Brazil
}

Abelardo de Souza Couto Júnior ${ }^{1}$, Juliana Lopes Jardim², Daniel Almeida de Oliveira ${ }^{3}$, Thiago Capilla Gobetti ${ }^{4}$, Arlindo José Freire Portes ${ }^{5}$, Rogério Neurauter ${ }^{6}$

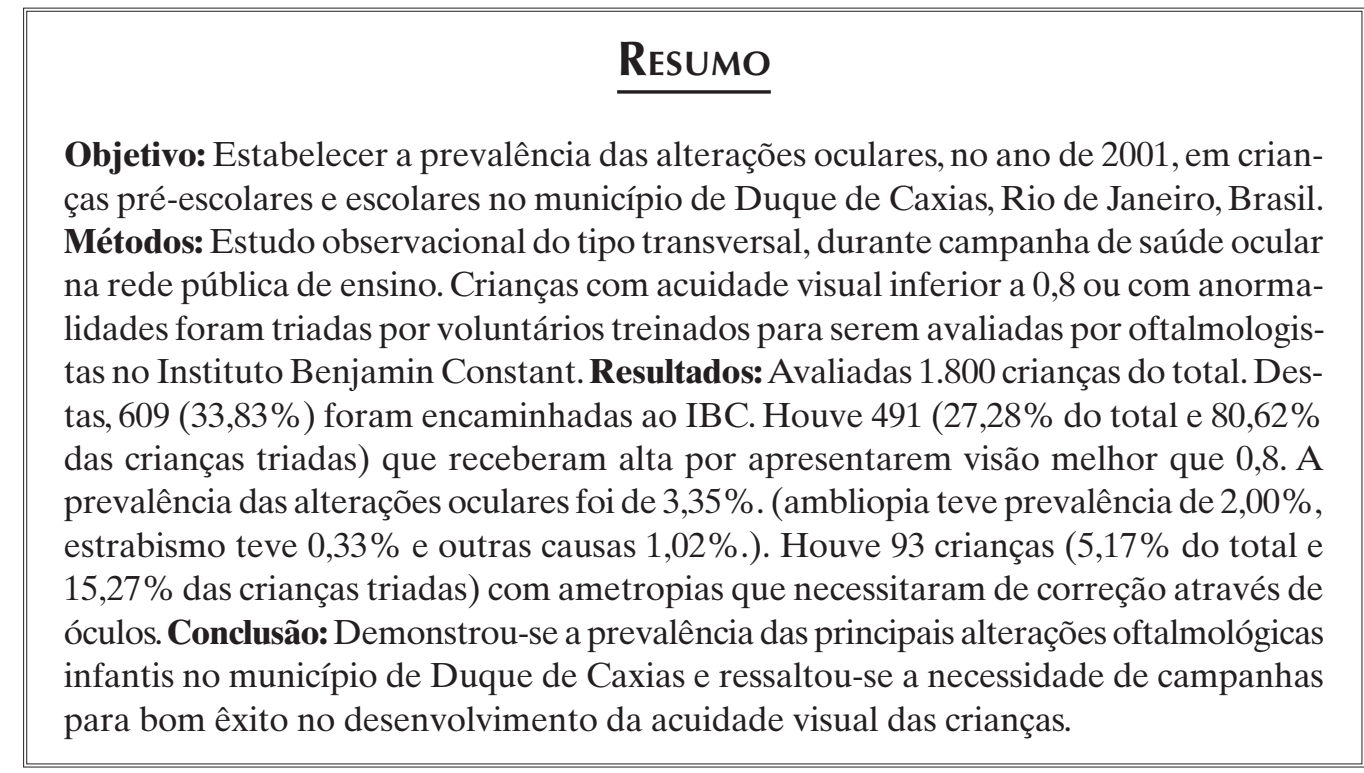

Descritores: Oftalmopatias;Ambliopia; Estrabismo; Saúde escolar;Promoção da saúde;

Pré-escolar; Criança

\footnotetext{
'Doutor, Professor Titular de Oftalmologia da Faculdade de Medicina de Valença; Coordenador da Residência Médica em Oftalmologia do Instituto Benjamin Constant - Rio de Janeiro (RJ), Brasil; Professor da Pós-Graduação em Oftalmologia da Pontifícia Universidade Católica PUC - Rio de Janeiro (RJ), Brasil;

${ }^{2}$ Médica Oftalmologista especialista em Glaucoma e Catarata pela Faculdade de Medicina da Universidade de São Paulo (HCFMUSP); Fellow em Glaucoma pelo New York Eye and Ear Infirmary - New York- EUA; Ex-residente do Instituto Benjamin Constant - Rio de Janeiro (RJ), Brasil;

${ }^{3,4}$ Residentes do Instituto Benjamin Constant - Rio de Janeiro (RJ), Brasil;

${ }^{5}$ Doutor, Professor Adjunto de Oftalmologia da Faculdade de Medicina Estácio de Sá - Rio de Janeiro (RJ), Brasil; Coordenador da Especialização Médica em Oftalmologia do Hospital Geral de Bonsucesso - Rio de Janeiro (RJ), Brasil; Diretor de Cursos da Sociedade Brasileira de Oftalmologia (SBO) - Rio de Janeiro (RJ), Brasil;

${ }^{6}$ Master Business Administration, Administração em Oftalmologia pela Universidade Federal de São Paulo - UNIFESP; Chefe do Departamento de Oftalmologia do Instituto Benjamim Constant - IBC - Rio de Janeiro (RJ), Brasil.
}

Trabalho realizado no Instituto Benjamin Constant - IBC - Rio de Janeiro (RJ), Brasil. 


\section{INTRODUÇÃO}

A s alterações oftalmológicas nas crianças em idade pré-escolar e escolar deveriam ser prioridade nos programas de saúde pública em oftalmologia, pois as mais sérias, como estrabismo, ametropias, anisometropias, cataratas congênitas, retinoblastomas, glaucoma, malformações, etc., podem levar à incapacidade visual e à cegueira e são passíveis de serem descobertas pela triagem e tratadas. Tal prioridade teria a finalidade de garantir o pleno desenvolvimento das potencialidades, $\mathrm{o}$ incremento no rendimento escolar e a participação consciente do indivíduo na sociedade ${ }^{(1-8)}$.

Aproximadamente $85 \%$ do processo de aprendizado passa pelo sentido da visão. A deficiência visual afeta de maneira negativa o processo de aprendizagem. Entretanto, estima-se que a maioria das crianças brasileiras em idade escolar nunca passou por exame oftalmológico. Isso decorre de dificuldades sócioeconômicas da família, de acesso a serviços especializados e da não detecção da alteração oftalmológica por pessoas não treinadas ${ }^{(1-3,9)}$.

Cerca de $20 \%$ das crianças em idade escolar apresentam algum transtorno da visão ${ }^{(10)}$. A investigação em exame de massa de problemas oculares em crianças por meio de oftalmologistas mostra-se muito dispendiosa e mesmo inexequível em saúde pública. Uma das alternativas viáveis é a triagem por pessoal não médico treinado e supervisionado em populações de crianças desde o ingresso na escola, para posterior avaliação por oftalmologistas ${ }^{(1-10)}$.

De acordo com uma estimativa para o ano de 2002 da Organização Mundial de Saúde (OMS) ${ }^{(11)}$, há quase 36,9 milhões de cegos no mundo. Aproximadamente dois terços deste cegos são por causas preveníveis e cerca de 1,4 milhão têm menos de quinze anos de idade. Das crianças portadoras de cegueira, 70 a $80 \%$ morrem durante os primeiros anos de vida, em consequência de doenças associadas ao seu comprometimento visu$\mathrm{al}^{(12-13)}$. Os países em desenvolvimento contribuem com quase a totalidade dos casos de cegueira potencialmente evitáveis ${ }^{(10-13)}$. A baixa visão apresenta uma prevalência cinco a sete vezes maior nos países pobres, se comparados aos ricos e mais de $90 \%$ dos casos de cegueira em menores de 16 anos são nos pobres ${ }^{(10)}$. Há, pela citada estimativa da $\mathrm{OMS}^{(11)}$, na região geográfica compreendida pelo Brasil, Barbados e Paraguai, quase 1,4 milhão de cegos e 7,6 milhões de pessoas com baixa visão. No Brasil existem 98 milhões de pessoas com algum tipo de deficiência visual, dos quais 80 milhões não têm acesso a qualquer tratamento ${ }^{(12-13)}$.
Para o combate a cegueira é necessário o conhecimento sobre a prevalência e o fator causal, que variam conforme condições geográficas e sócioeconômicas ${ }^{(11)}$. O Brasil, pelas suas dimensões continentais e diferenças econômicas e culturais nos diversos estados, demanda avaliação ampla para o estabelecimento de programas de prevenção das causas de comprometimento visual infantil ${ }^{(3)}$. Quanto mais tardio é o diagnóstico das alterações oculares da infância, pior é o prognóstico e mais graves são as sequelas ${ }^{(10)}$.

O objetivo deste estudo foi estabelecer a prevalência das alterações oculares em crianças de idade pré-escolar e escolar no município de Duque de Caxias no ano de 2001, a fim de demonstrar a importância das campanhas de prevenção de cegueira para a análise da prevalência e prevenção das causas de cegueira e orientação de política comunitária de saúde ocular.

\section{MÉTodos}

Foi realizado um estudo observacional do tipo transversal em 2001, durante campanha de saúde ocular no município de Duque de Caxias, Rio de Janeiro, em crianças da rede pública de ensino. Foram realizados exames de triagem ocular por voluntários treinados, que consistiam na aferição da acuidade visual através da tabela de Snellen e avaliação ocular externa com lupa e lanterna em crianças que estudavam na rede pública daquele município, na pré-escola e escola até a quarta série, não importando a idade. A campanha "Projeto Sul América de Saúde Ocular" contou com a participação conjunta do Instituto Benjamin Constant - IBC, Secretaria Municipal de Educação de Duque de Caxias, e as organizações sem fins lucrativos, Helen Keller Worldwide e Pró-Natura. Ao final da campanha foram doados óculos às crianças que necessitaram de correção para suas ametropias. Foi considerado como ambliopia a acuidade visual corrigida de 0,8 ou menos no pior olho e anisometropia como sendo a diferença de 2,00 dioptrias esféricas ou o equivalente esférico entre os dois olhos $^{(3,5-6)}$. As crianças com AV menor ou igual a $0,8 \mathrm{ou}$ com alguma anormalidade foram encaminhadas para exame completo pelos oftalmologistas do IBC.

\section{Resultados}

Voluntários treinados triaram 1.800 crianças no município de Duque de Caxias. Destas, 609 (33,83\%) foram encaminhadas ao IBC para reavaliação. Das 609 
crianças, 491 (27,28\% do total e $80,62 \%$ das crianças triadas) receberam alta após exames oftalmológicos por apresentarem visão melhor que 0,8 em um ou ambos os olhos. A tabela 1 mostra a prevalência das alterações oculares, que foram observadas em 61 crianças $(3,35 \%$ do total). Houve 36 casos de ambliopia, 6 casos de estrabismo manifesto e 19 casos de outras alterações. A prevalência de ambliopia (2,00\%) se destacou das demais. A maior causa de ambliopia foi a refrativa $(0,72 \%)$, seguido pela secundária ao estrabismo $(0,55 \%)$. O estrabismo manifesto teve como prevalência $0,33 \%$. As outras causas foram responsáveis por uma prevalência de $1,02 \%$. Detectou-se 93 crianças $(5,17 \%$ do total e $15,27 \%$ das crianças triadas) com ametropias que necessitaram de correção através de óculos.

\section{DiscussÃo}

A amostra populacional estudada, de pré-escolares e escolares do município de Duque de Caxias, é heterogênea, com algumas crianças advindas de zona rural, onde o acesso à avaliação oftalmológica é mais difícil, e outras de área urbana. Não estão disponíveis dados sobre evasão escolar para o ano de 2001 naquele municí- pio, mas pôde-se supor que algumas crianças portadoras de acuidade visual incompatível com o ensino público ministrado tenham abandonado a escola. Tal fato geraria subestimação das reais prevalências para aquela população. ${ }^{(3)}$

A prevalência da ambliopia varia de 1 a $8 \%$ na literatura $^{(1-3,5,8-16)}$. Scarpi ${ }^{(6)}$, em 1977, relatou uma prevalência em estudantes de São Paulo de 4,07\%. Neurauter ${ }^{(17)}$, em 2001, relatou uma prevalência em préescolares e escolares dos bairros de Copacabana e Urca - RJ de 1,39\%. Schimiti(7) , em 2001, relatou em Ibiporã uma prevalência de $1,76 \%$. Couto $\mathrm{Jr} .^{(3)}$, nas favelas do Alto da Boa Vista, encontrou prevalência de 2,00\%. Lopes $^{(1)}$, em 2002, encontrou 3,6\% em alunos de escolas estaduais e $5,9 \%$ em alunos de escolas particulares da cidade de Londrina. Beer ${ }^{(5)}$, para São Caetano, relatou uma prevalência hipotética de $2,8 \%$. Portes ${ }^{(15)}$, na população de 3 a 6 anos assistida pelo Programa de Saúde da Família (PSF) na Lapa - Rio de Janeiro, em 2005 , encontrou prevalência de $8 \%$. Jeveaux ${ }^{(16)}$, em crianças de 3 a 6 anos assistidas pelo PSF no Morro do Alemão (Rio de Janeiro), em 2006, encontrou prevalência de $4,1 \%$. Nas crianças de Duque de Caxias, este estudo encontrou uma prevalência de $2,00 \%$, o que não fugiu ao padrão descrito.

Tabela 1

Prevalência das alterações oculares em crianças pré-escolares e escolares em 2001, no município de Duque de Caxias, Rio de Janeiro, Brasil

\begin{tabular}{|c|c|c|c|}
\hline Alterações oculares & Número & $\begin{array}{c}\text { Prevalência em relação } \\
\text { à população de } 1.800 \text { crianças }\end{array}$ & $\begin{array}{l}\text { \% em relação ao total } \\
\text { de oftalmopatias (61) }\end{array}$ \\
\hline Ambliopia & 36 & $2,00 \%$ & $59,02 \%$ \\
\hline Refrativa & 13 & $0,72 \%$ & $21,31 \%$ \\
\hline Secundária ao estrabismo & 10 & $0,55 \%$ & $16,39 \%$ \\
\hline Etiologia desconhecida & 1 & $0,05 \%$ & $1,64 \%$ \\
\hline Anisometropia & 2 & $0,11 \%$ & $3,28 \%$ \\
\hline Estrabismo manifesto & 6 & $\mathbf{0 , 3 3} \%$ & $\mathbf{9 , 8 4 \%}$ \\
\hline Esotropia & 4 & $0,22 \%$ & $6,56 \%$ \\
\hline Exotropia & 2 & $0,11 \%$ & $3,28 \%$ \\
\hline Catarata congênita & $\mathbf{1}$ & $\mathbf{0 , 0 5} \%$ & $1,64 \%$ \\
\hline Coriorretinite & 5 & $0,27 \%$ & $8,20 \%$ \\
\hline Binocular & 1 & $0,05 \%$ & $1,64 \%$ \\
\hline Monocular & 4 & $0,22 \%$ & $6,56 \%$ \\
\hline Ptose palpebral & $\mathbf{1}$ & $\mathbf{0 , 0 5} \%$ & $1,64 \%$ \\
\hline Conjuntivite & 2 & $0,11 \%$ & $3,28 \%$ \\
\hline Escavação aumentada & 6 & $\mathbf{0 , 3 3} \%$ & $\mathbf{9 , 8 4} \%$ \\
\hline Atrofia óptica & 2 & $0,11 \%$ & $3,28 \%$ \\
\hline Nistagmo & 1 & $\mathbf{0 , 0 5} \%$ & $1,64 \%$ \\
\hline Leucoma corneano pós-trauma & 1 & $\mathbf{0 , 0 5} \%$ & $1,64 \%$ \\
\hline Total & 61 & $3,35 \%$ & $100 \%$ \\
\hline
\end{tabular}


A maior parte dos trabalhos referem uma prevalência de estrabismo em populações gerais de 1 a $4 \%^{(1,18-20)}$. Macchiaverni ${ }^{(4)}$, em 1979 , encontrou em Paulínea a prevalência de $1,58 \%$. Neurauter ${ }^{(17)}$ encontrou $0,69 \%$ após exames de 1144 escolares em dois bairros da Zona Sul da cidade do Rio de Janeiro. Schimiti ${ }^{(7)}$, em 2001, encontrou em Ibiporã, 0,84\%. Couto Jr. ${ }^{(3)}$, em 2001, encontrou-se $1,72 \%$ de prevalência nas favelas do Alto da Boa Vista. Lopes ${ }^{(1)}$, em 2002, achou em Londrina, $0,46 \%$ em alunos de escolas estaduais e $2,4 \%$ das escolas particulares. Beer ${ }^{(5)}$, em 2003, encontrou em São Caetano do Sul, 1,78\%. Garcia ${ }^{(20)}$, em 2004, encontrou, nos estudantes de Natal, 2,9\%. Portes ${ }^{(15)}$, em 2005, encontrou $2,7 \%$ na Lapa. Jeveaux ${ }^{(16)}$ observou $1,8 \%$ em 2006 no Morro do Alemão. O presente estudo encontrou em Duque de Caxias uma prevalência de 0,33\%, um pouco abaixo do que seria esperado. Quanto ao tipo de estrabismo, a literatura apresenta a esotropia como de duas a três vezes mais frequentes que a exotropia ${ }^{(5,7)}$, com algumas populações como exceção ${ }^{(3,20)}$. Neste estudo, a esotropia $(0,22 \%)$ foi duas vezes mais frequente que a exotropia $(0,11 \%)$. Uma avaliação complementar posterior se faz necessária para a confirmação desta prevalência menor de estrabismo, mas talvez particularidades na população estudada possam explicá-la ${ }^{(1,3,20)}$.

Em relação aos outros tipos de alterações oculares, o presente estudo demonstrou uma prevalência de $1,02 \%$. A prevalência dessas alterações varia bastante na literatura e a maneira de classificá-las prejudica um pouco a comparação. Em um estudo similar, Schimiti ${ }^{(7)}$ encontrou cerca de $4,03 \%$, com $1,93 \%$ de coriorretinite e $0,86 \%$ de catarata. Neurauter ${ }^{(17)}$ encontrou $5,31 \%$, destes $0,17 \%$ de catarata congênita, $0,08 \%$ de catarata traumática e $0,08 \%$ de doença retiniana macular. Couto Jr. ${ }^{(3)}$ encontrou uma prevalência de $1,11 \%$, sendo $0,22 \%$ de catarata e $0,17 \%$ de coriorretinite. Este estudo encontrou uma prevalência de $0,27 \%$ de coriorretinite, mas apenas $0,05 \%$ de catarata congênita. Várias causas podem ser aventadas para as variações, sendo necessário estudos posteriores ${ }^{(3)}$.

Neste estudo encontrou-se a prevalência de ametropias com necessidade de correção por óculos em $5,17 \%$ da população de pré-escolares e escolares. Os dados da literatura sobre tal prevalência são variáveis, desde $2,4 \%^{(1)}, 3,50 \%^{(3)}, 3,6 \%^{(1)}, 4,56 \%^{(7)}, 4,98 \%^{(17)}$, $10,8 \%^{(15)}, 14,11 \%^{(5)}$ a até $15,8 \%{ }^{(16)}$.

Em saúde pública, a triagem mostra-se necessária, pois uma grande parcela de crianças chegam à escola sem nunca ter passado por um exame oftalmológico. Em torno de $15 \%$ das crianças da primeira série escolar possuem alguma alteração visual e apenas $20 \%$ dessas crianças tem acompanhamento médico ${ }^{(1,5-7,10,20)}$. Todas as crianças deste estudo que tiveram necessidade estão sendo acompanhadas no serviço de oftalmologia no IBC.

$\mathrm{O}$ atraso ou até mesmo o não tratamento das crianças portadoras de ambliopia piora seriamente o prognóstico visual, principalmente na associação ambliopia e estrabismo ${ }^{(4-5,10)}$. Com diagnóstico e tratamento precoces, a ambliopia é reversível na maior parte dos $\operatorname{casos}^{(9)}$. Porém, a maioria se apresenta para tratamento após a idade de seis anos, quando o mesmo é menos eficaz e a aceitação da terapêutica é mais difícil ${ }^{(2,8-9)}$. As crianças deste estudo diagnosticadas com ambliopia eram todas de idade acima de seis anos, por serem pré-escolares e escolares. Isto demonstra a necessidade de ações que abordem as crianças que não estão ainda na escola, como fez, por exemplo, Beer em São Caetano do Sul, durante a vacinação de poliomielite (crianças menores que cinco anos) ${ }^{(5)}$.

A triagem através da medida da acuidade visual pela tabela de Snellen é um método de fácil aplicação e de baixo custo ${ }^{(1,3,5,7,10,20,21)}$. A sua utilização por pessoal treinado deveria ser amplamente estimulada, principalmente em associação ao exame externo com lupa e lanterna, em populações mais susceptíveis, como as crianças menores e as que não contam com serviço oftalmológico. Tal prática mostra-se bastante eficaz para a identificação precoce das afecções oculares e para permitir medidas preventivas e terapêuticas precoces e eficazes $^{(1,3,21-23)}$.

\section{Conclusão}

Demonstrou-se a prevalência das principais oftalmopatias infantis no município de Duque de Caxias. Faz-se mister ressaltar a necessidade de outras investigações complementares dessa mesma linha de pesquisa na população pré-escolar e escolar, a fim de identificar precocemente as causas de ambliopia, para um tratamento mais eficaz das alterações oftalmológicas e um bom êxito no desenvolvimento da acuidade visual das crianças, assim como poder extrapolar os dados para a população em geral.

\section{Abstract}

Purpose: To establish the prevalence of the ametropias and eye diseases, in the year 2001, within a preschool and school children in the city of Duque de Caxias, Rio de Janeiro, Brazil. Methods: Transversal observational study during an ocular health campaign in public education schools. 
The children that have shown visual acuity lower than 0.8 or other abnormally were referred by trained volunteers to evaluation by ophthalmologists from the Benjamin Constant Institute. Results: From the 1.800 children who were examined, $609(33.83 \%)$ were referred to the ophthalmologic examination. There were 491 children (27.28\% from total and $80.62 \%$ from referred) that were dismissed for presenting visual acuity better than 0.8. The Eye diseases prevalence was $3.35 \%$ (amblyopia was $2.00 \%$, manifest strabismus was $0.33 \%$ and others causes was $1.02 \%$ ). There was 93 children (5.17 from total and $15.27 \%$ from referred) that had refractive errors and needed glasses. Conclusion: It was shown the prevalence of the main ophthalmologic children disorders. It also points out the need of ocular health campaigns thus achieve remarkably the development of the children visual acuity.

Keywords: Eye diseases; Amblyopia; Strabismus; School health; Health promotion; Child, preschool; Child

\section{ReferêNCIAS}

1. Lopes GJA, Casella AMB, Chuí CA. Prevalência de acuidade visual reduzida nos alunos da primeira série do ensino fundamental das redes pública estadual e privada de Londrina-PR, no ano de 2000. Arq Bras Oftalmol. 2002; 65(6): 659-64 .

2. Bechara SJ, Kara-José N. Deteç̧ão e tratamento de pacientes amblíopes na cidade de São Paulo, SP (Brasil). Rev Saúde Pública. 1987; 21(4): 326-30.

3. Couto Jr AS, Pinto GR, Oliveira DA, Holzmeister D, Portes ALF, Neurauter R, Portes AJF. Prevalência das ametropias e oftalmopatias em crianças pré-escolares e escolares em favelas do Alto da Boa Vista, Rio de Janeiro, Brasil. Rev Bras Oftalmol. 2007; 66(5): 304-8.

4. Macchiaverni Filho N, Kara-José N, Rueda G, Pereira VL, Costa MN, Rangel FF, Fávero M. Levantamento oftalmológico em escolares de primeira a quarta séries do primeiro grau na cidade de Paulínea, São Paulo. Arq Bras Oftalmol. 1979; 42(6): 289-94.

5. Beer SMC, Scarpi MJ, Minello AA. Achados oculares em crianças de zero a seis anos de idade, residentes na cidade de São Caetano do Sul, SP. Arq Bras Oftalmol. 2003; 66(6): 839-45.

6. Scarpi MJ, Kara-José N, Taiar A. Incidência de ambliopia em 1400 escolares da cidade de São Paulo, em 1975. Arq Bras Oftalmol. 1977; 40(1): 16-23.

7. Schimiti RB, Costa VP, Gregui MJF, Kara-José N, Temporini ER. Prevalence of refractive errors and ocular disorders in preschool and schoolchildren of Ibiporã-PR, Brazil (1989 to 1996). Arq Bras Oftalmol. 2001; 64(5): 379-84.

8. Kara José N, Carvalho KMM, Caldato R, Pereira VL, Oliveira AMND, Fonseca Neto JC. Atendimento de amblíopes e prevalência na população pré-escolar, Campinas, São Paulo, Brasil. Bol Oficina Sanit Panam. 1984; 96(1): 31-7.
9. Trigueiro SA, Lucena A, Dickson A, Tavares S, Ventura LO. Aderência ao tratamento da ambliopia em centro oftalmológico de referência do grande Recife - Pernambuco - Brasil. An Fac Med Univ Fed Pernamb. 1999; 44(2): 118-21.

10. Albuquerque RC, Alves JGB. Afecções oculares prevalentes em crianças de baixa renda atendidas em um serviço oftalmológico na cidade do Recife - PE, Brasil. Arq Bras Oftalmol 2003;66(5):831-4.

11. Resnikoff S, Pascolini D, Etya'ale D, Kocur I, Pararajasegaram R, Pokharel GP, Mariotti SP. Global data on visual impairment in the year 2002. Bull World Health Organ. 2004; 82(11): 844-51.

12. Oliveira PR. Causas da cegueira na infância. Arq Bras Oftalmol. 1992; 55(4): 172-5.

13. Brito PR, Veitzman S. Causas de cegueira e baixa visão em crianças. Arq Bras Oftalmol. 2000; 63(1): 49-54.

14. Olivier M, Nawratzki I. Screening of pre-school children for ocular anomalies. II - Amblyopia. Prevalence and therapeutic results at different ages. Br J Ophthalmol. 1971; 55(7):467-71.

15. Portes AJF, Portes ALF, Bonfadini CG, Shinzato FA, Pecego MG, Silva ASSB. Prevenção à cegueira em crianças de três a seis anos assistidas pelo Programa de Saúde da Família Lapa, Rio de Janeiro. Rev Bras Oftalmol. 2007; 66(3):155-9.

16. Jeveaux G, Portes AJF, Couto Júnior AS, Shinzato F. Prevenção à cegueira em crianças de 3 a 6 anos assistidas pelo Programa de Saúde da Família (PSF) do Morro do Alemão - Rio de Janeiro. Rev Bras Oftalmol. 2008; 67(5):226-230.

17. Neurauter R, Bellini AT, Couto-Júnior AS. Saúde ocular de oré-escolares dos bairros de Copacabana e Urca - Rio de Janeiro. Rev Bras Oftalmol. 2001; 60( ): 199-203.

18. Abrahamsson M, Fabian G, Sjöstrand J. Refraction changes in children developing convergent or divergent strabismus. Br J Ophthalmol. 1992; 76(12):723-7.

19. Preslan MW, Novak A. Baltimore Vision Screening Project. Ophthalmology. 1996; 103(1):105-9.

20. Garcia CAA, Sousa AB, Mendonça MBM, Andrade LL, Orefice F. Prevalence of strabismus among students in Natal/RN Brazil. Arq Bras Oftalmol. 2004;67(5):791-4.

21. Couto Jr AS. Considerações preliminares sobre a identificação das deficiências visuais em pré-escolares e escolares [monografia]. Rio de Janeiro; Instituto Benjamin Constant; 1992. [Apresentada ao Curso de Pós Graduação em Oftalmologia Clínica e Cirúrgica. Instituto Benjamin Constant].

22. Temporini ER. Níveis de prevenção de problemas oftalmológicos: Propostas de investigação. Rev Bras Oftalmol. 1993; 52(4): 49-52.

23. Vieira C, Rodrigues MLV. Prevenção da cegueira nas escolas rurais da região de Santa Bárbara D’Oeste - SP. Rev Bras Oftalmol. 1995; 54(2): 43-7.

Abelardo de Souza Couto Júnior
Av. Nossa Senhora de Copacabana, $\mathbf{n}^{\circ} 1120$
apto. 901
CEP $22020-001$ - Copacabana - RJ
Tel: (21)-2521.0645
E-mail: soluc@ig.com.br

